\title{
Solubility isotope effects in aqueous solutions of methane
}

\author{
Z. Bacsik, ${ }^{\text {a) }}$ J. N. Canongia Lopes, ${ }^{\text {b) }}$ M. F. Costa Gomes, ${ }^{c)}$ G. Jancsó, ${ }^{\text {d) }}$ \\ J. Mink, ${ }^{\text {a),e),f) }}$ and A. A. H. Pádua ${ }^{\text {c) }}$ \\ Centro de Química Estrutural, Instituto Superior Técnico, 1049-001 Lisboa, Portugal
}

(Received 4 December 2001; accepted 28 March 2002)

\begin{abstract}
The isotope effect on the Henry's law coefficients of methane in aqueous solution (H/D and ${ }^{12} \mathrm{C} /{ }^{13} \mathrm{C}$ substitution) are interpreted using the statistical mechanical theory of condensed phase isotope effects. The missing spectroscopic data needed for the implementation of the theory were obtained either experimentally (infrared measurements), by computer simulation (molecular dynamics technique), or estimated using the Wilson's $G F$ matrix method. The order of magnitude and sign of both solute isotope effects can be predicted by the theory. Even a crude estimation based on data from previous vapor pressure isotope effect studies of pure methane at low temperature can explain the inverse effect found for the solubility of deuterated methane in water. (c) 2002 American Institute of Physics. [DOI: 10.1063/1.1480012]
\end{abstract}

\section{INTRODUCTION}

The study of the isotope effects on Henry's law coefficients in aqueous solutions can be traced to a number of applications in various fields ranging from biophysics to geochemistry. Isotope effects are often used to obtain information about reaction mechanisms and pathways, providing valuable data about reactions in a mechanistic sense, including the structure of the transition state. ${ }^{1-3}$ Effects concerning isotopically substituted molecules (isotopomers) of methane are of great interest to the petroleum industry as the knowledge of the concentration of each isotopomer contributes to the characterization of petroleum as gas reservoirs. ${ }^{1}$ The same effects, especially on the Henry's law coefficients, can also play an important role in biological studies., ${ }^{2,3}$

The available experimental data on the isotope effect on the solubility of gases in liquids (mainly in water) have been recently reviewed. ${ }^{4}$ From the theoretical point of view solubility isotope effects in aqueous solutions constitute excellent tests of the application and generalization of the statistical mechanical theory of isotope effects in condensed phases. ${ }^{5-7}$

In a previous paper, the solubility isotope effect in aqueous solutions of methane (H/D substitution) has been measured as a function of temperature at nearly atmospheric pressures. ${ }^{8}$ The Henry's law coefficients obtained allowed the calculation of other thermodynamic properties characteristic of the solution process, namely the standard Gibbs en-

a) Also with Institute of Isotope and Surface Chemistry, Chemical Research Center of the Hungarian Academy of Sciences, P.O. Box 77, H-1525 Budapest, Hungary.

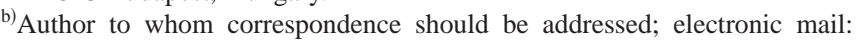
jnlopes@ist.utl.pt

${ }^{c}$ Present address: Laboratoire de Thermodinamique des Solutions Polymeres, UMR 6003, 24 Avenue des Landais, 63177 Aubiere cedex, France.

${ }^{d)}$ Present address: KFKI Atomic Energy Research Institute, P.O. Box 49, 1525 Budapest, 114 Hungary.

e) Also with Department of Analytical Chemistry, University of Veszprém, P.O. Box 158, H-8201 Veszprém, Hungary.

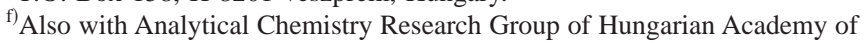
Sciences, P.O. Box 158, H-8201 Veszprém, Hungary. ergy, enthalpy, and heat capacity of solution. It was also observed that the Henry's law coefficients for tetradeuteriomethane in water are higher than those of methane in water (at $298 \mathrm{~K}, \mathrm{CD}_{4}$ is less soluble in water than $\mathrm{CH}_{4}$ by approximately $1.5 \%$ ). In other words, deuterium substitution results in an inverse solubility isotope effect in aqueous solutions of methane, ${ }^{5}$ a result contrasting with other solubility isotope effect data in aqueous solutions. In the case of helium ${ }^{9}$ and a number of diatomic molecules ${ }^{10,11}$ dissolved in water, the heavier isotopic species is always more soluble than the lighter one. The same normal effect was observed in the case of ${ }^{12} \mathrm{CH}_{4}$ and ${ }^{13} \mathrm{CH}_{4}$ in water (the solubility of ${ }^{13} \mathrm{CH}_{4}$ in water is higher than that of ${ }^{12} \mathrm{CH}_{4}$ by $0.06 \%$ at $\left.293 \mathrm{~K}\right){ }^{12}$

In the next sections both inverse and normal solubility isotope effects in aqueous solutions of methane (H/D and ${ }^{12} \mathrm{C} /{ }^{13} \mathrm{C}$ substitutions, respectively) will be interpreted within the framework of the statistical mechanical theory of condensed phase isotope effects. The discussion will focus solely on solutes undergoing isotopic substitition (the effect of $\mathrm{H} / \mathrm{D}$ substitution on the solvent is not considered).

\section{STATISTICAL MECHANICAL THEORY OF CONDENSED PHASE ISOTOPE EFFECTS}

The solubility of gases in liquids can be regarded as a particular example of liquid-vapor equilibria in binary mixtures where one of the components (the solute) is already above its critical temperature. Therefore, the effect of isotopic substitution on the Henry's law coefficients can be interpreted using a generalized version of the statistical theory of vapor pressure isotope effects (VPIEs) as developed originally by Bigeleisen. ${ }^{13}$ The application of the VPIE theory is even more straightforward in the present case since the ratio of the Henry's law coefficients of the two isotopically substituted molecules is equal to the ratio of the so-called reduced partition function ratios (RPFRs) ${ }^{14}$ of the liquid and gas phases 


$$
\frac{K_{1}}{K_{2}}=\frac{f_{\text {liq }}}{f_{\text {gas }}},
$$

whereas in the case of vapor pressure data, the experimental pressure results have to be corrected to yield the fugacity of each species ${ }^{13}\left(K_{1}\right.$ and $K_{2}$ are the Henry's law coefficients for the lighter and heavier isotopic species, respectively, $f$ is the reduced partition function ratio, and the subscripts liq and gas refer to the liquid and gas phases, respectively).

The RPFR both in the liquid and vapor phases can be expressed in terms of the frequencies of all $3 n$ vibrational modes of each isotopomer ( $n$ is the number of atoms in the molecule). These can be evaluated using a single-cell model to describe the liquid phase and assuming the harmonic oscillator approximation for each vibrational mode. ${ }^{15}$ In the present case, the methane molecules are described as spherical tops acting as rigid rotors with unhindered translational and rotational motions in the gas phase.

The RPFR of the gas phase can be written as

$$
f_{\text {gas }}=\prod_{i=1}^{9} \frac{u_{i, 2}}{u_{i, 1}} \exp \left(\frac{u_{i, 1}-u_{i, 2}}{2}\right)\left(\frac{1-\exp \left(-u_{i, 1}\right)}{1-\exp \left(-u_{i, 2}\right)}\right) f_{\text {ncrot }},
$$

where $u_{i}=h c \nu_{i} / k_{b} T$ ( $\nu_{i}$ is the harmonic frequency of the $i$ th normal mode in $\mathrm{cm}^{-1}, h, c$, and $k_{b}$ are the Planck, speed of light, and Boltzmann constants, respectively), and $f_{\text {ncrot }}$ is the contribution to the reduced partition function ratio arising from nonclassical rotation in the gas phase. The product in Eq. (2) runs over the nine internal vibrational modes of the methane molecule in the gas phase: the nondegenerate $(A)$ symmetric stretching, the doubly degenerate $(E)$ symmetric bending, and the triply degenerate $(T)$ antisymmetric stretching and bending normal modes. The $f_{\text {ncrot }}$ term can be readily evaluated for spherical tops with known moments of inertia ${ }^{16}$ (see discussion below).

The RPFR of the liquid phase is given by

$$
f_{\text {liq }}=\prod_{i}^{15} \frac{u_{i, 2}}{u_{i, 1}} \exp \left(\frac{u_{i, 1}-u_{i, 2}}{2}\right)\left(\frac{1-\exp \left(-u_{i, 1}\right)}{1-\exp \left(-u_{i, 2}\right)}\right),
$$

where the product now runs over the nine internal vibrational modes plus the triply degenerate hindered translational and triply degenerate hindered rotational modes of the methane molecules in the liquid phase.

According to Eqs. (1)-(3), the only data needed to evaluate theoretically the ratio of the Henry's law coefficients between a pair of methane isotopomers are four sets of spectroscopic data: (i) the frequencies of the nine vibrational modes of methane in the gas phase; (ii) the internal vibrational frequencies for methane dissolved in water; (iii) the hindered translational frequency; and (iv) and the hindered rotational frequency of methane in water. These four sets need to be known only for one of the three isotopic methane molecules, the spectroscopical data needed for the other two isotopomers can be generated using the Wilson's FG matrix method. ${ }^{17}$

The set of frequencies (i) can be easily obtained: both infrared (IR) and Raman spectra of pure gaseous methane are well determined and the corresponding $F$ and $G$ matrices are available in the literature ${ }^{18}$ (the vapor phase in equilibrium with the aqueous solution is not constituted solely by methane, but at room temperature and low pressure the interactions with the minute amounts of water present will have a negligible effect in the frequency values of the internal modes of methane).

The situation is rather more complicated in the case of the frequency sets (ii), (iii), and (iv). There are no spectroscopic data available in the literature on aqueous solutions of methane, a situation easily explained by the poor solubility of methane in water (solute mole fractions of around $10^{-5}$ ), requiring the use of spectroscopic techniques of very high accuracy.

\section{EXPERIMENT}

In order to obtain the missing sets of spectroscopic data three procedures were implemented: (i) the antisymmetric stretching and bending internal frequencies were accessed experimentally by IR spectroscopy; (ii) the symmetric stretching and bending frequencies were estimated using the measured values of the antisymmetric vibrational frequencies plus the ratios between symmetric and antisymmetric vapor-liquid frequency shifts observed for pure methane; and (iii) the external frequencies (hindered translation and rotation) were estimated using molecular dynamics simulation methods.

\section{A. Spectroscopic measurements}

The infrared spectra of solutions of $\mathrm{CH}_{4}(99.995 \mathrm{v} / \mathrm{v} \%$, Messer) in $\mathrm{D}_{2} \mathrm{O}$ (99.87 at. \% deuterium content, Isotop, Moscow) were measured with a Bio-Rad (Digilab) FTS-175C system, equipped with a liquid nitrogen cooled broadband $\mathrm{HgCdTe}$ (MCT) detector. The spectral resolution was $4 \mathrm{~cm}^{-1}$ and 1024 scans were accumulated. Due to the high throughput and good quality of the dynamical alignment, high signal/noise spectra could be recorded with rather dilute $\mathrm{D}_{2} \mathrm{O}$ solution samples. The use of $\mathrm{D}_{2} \mathrm{O}$ instead of normal water prevented the overlap between the $\mathrm{CH}_{4}$ absorption bands [degenerate antisymmetric stretching $\left(\nu_{3}\right)$ and degenerate antisymmetric bending mode $\left.\left(\nu_{4}\right)\right]$ and the strong absorption bands of the solvent. The same reason precluded the use of $\mathrm{CD}_{4}$ in heavy water. Normal aqueous solutions of $\mathrm{CD}_{4}$ could be studied in principle but that would imply the use of a less readily available substance $\left(\mathrm{CD}_{4}\right)$ without any significant benefit in terms of precision or subsequent interpretation of the results.

For the room temperature measurements, $\mathrm{CaF}_{2}$ liquid cells with $0.1-1 \mathrm{~mm}$ path lengths were used. For the "low" temperature studies $\left(6^{\circ} \mathrm{C}\right)$ a vacuum-tight low-temperature cell fitted with $\mathrm{AgCl}$ windows and a spacer $0.25 \mathrm{~mm}$ thick was used. The temperature was established using a SPECAC 20-100 temperature controller and a commercially available SPECAC 21-000 low-temperature Dewar cell. The temperature stability is better than $0.2^{\circ} \mathrm{C}$, with an accuracy of $1{ }^{\circ} \mathrm{C}$. A $10 \mathrm{~cm}$ long gas cell was used to record the spectrum of gaseous methane with a resolution of $0.5 \mathrm{~cm}^{-1}$.

Since the solubility of $\mathrm{CH}_{4}$ in $\mathrm{D}_{2} \mathrm{O}$ is very low at room temperature, there are two ways to increase the amount of $\mathrm{CH}_{4}$ in the IR beam path. 


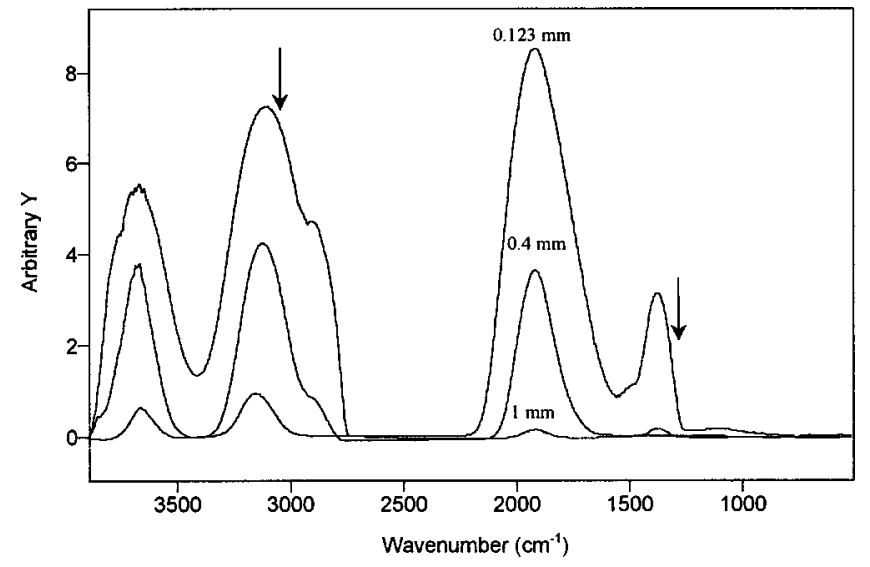

FIG. 1. Single beam spectra of $\mathrm{D}_{2} \mathrm{O}$ at different cell path lengths: $\mathrm{CaF}_{2}$ cells of $1,0.4$, and $0.123 \mathrm{~mm}$ are depicted. The positions marked with an arrow refer to the regions where the antisymmetric stretching and bending frequencies of methane $\left(\nu_{3}\right.$ and $\left.\nu_{4}\right)$ are to be found.

One possibility is to increase the cell path length. Preliminary single beam spectra of pure $\mathrm{D}_{2} \mathrm{O}$ in cells fitted with $\mathrm{CaF}_{2}$ windows and variable path length (Fig. 1) indicate that the degenerate antisymmetric stretching $\left(\nu_{3}\right)$ band of methane around $3016 \mathrm{~cm}^{-1}$ can be detected with cells of 1 or 0.4 $\mathrm{mm}$ path lengths. However, with these large path lengths no transparent window exists around $1305 \mathrm{~cm}^{-1}$, preventing the detection of the degenerate antisymmetric bending mode $\left(\nu_{4}\right)$ of $\mathrm{CH}_{4} \cdot \mathrm{A} \mathrm{D}_{2} \mathrm{O}$ sample with $0.123 \mathrm{~mm}$ path length has a broad window in the $1305 \mathrm{~cm}^{-1}$ region, adequate to record the $\nu_{4}$ band, but it was found that the intensity of that band is very close to the noise level. Finally, it was concluded that a $\mathrm{D}_{2} \mathrm{O}$ sample with $0.25 \mathrm{~mm}$ path length is still transparent enough at $1305 \mathrm{~cm}^{-1}$ to enable detection of the spectral features in that region and thick enough to have a measurable amount of dissolved methane in the infrared beam.

The second way to enhance the absorption of $\mathrm{CH}_{4}$ is to increase its concentration, e.g., to increase its solubility in $\mathrm{D}_{2} \mathrm{O}$ by cooling the solution. Since the $\mathrm{CaF}_{2}$ windows are very sensitive to temperature changes, a standard waterresistant $\mathrm{AgCl}$ windowed low temperature cell was used with $0.25 \mathrm{~mm}$ path length (the correct thickness according to the preliminary runs). A temperature of $6{ }^{\circ} \mathrm{C}$ was chosen in order to increase the solubility of $\mathrm{CH}_{4}$ in $\mathrm{D}_{2} \mathrm{O}$ as compared to the solubility at room temperature (the solubility is almost two times higher at $6{ }^{\circ} \mathrm{C}$ than at $25^{\circ} \mathrm{C}$ and the $\mathrm{D}_{2} \mathrm{O}$ is still liquid).

The double beam spectra of solutions of $\mathrm{CH}_{4}$ in $\mathrm{D}_{2} \mathrm{O}$ are shown in Fig. 2. The signal/noise ratio is excellent and the weak bands $\left(\nu_{3}\right.$ and $\left.\nu_{4}\right)$ on the slope of the $\mathrm{D}_{2} \mathrm{O}$ absorption can be clearly detected at 3016.5 and $1304.7 \mathrm{~cm}^{-1}$. Better spectral features can be obtained after baseline correction and ordinate expansion (Fig. 3). Since we are interested in the vapor-liquid frequency shifts, the IR active fundamentals of gaseous $\mathrm{CH}_{4}$ were measured simultaneously using the same spectrometer. The band origins of the $Q$ branches are tabulated in Table I, along with the results obtained in aqueous solution.

It can be seen in Fig. 3 that the intensity of the $\nu_{3}$ band is almost two times higher than that for the $\nu_{4}$ mode. The noise level near $\nu_{4}$ is about 0.0008 absorbance units and the
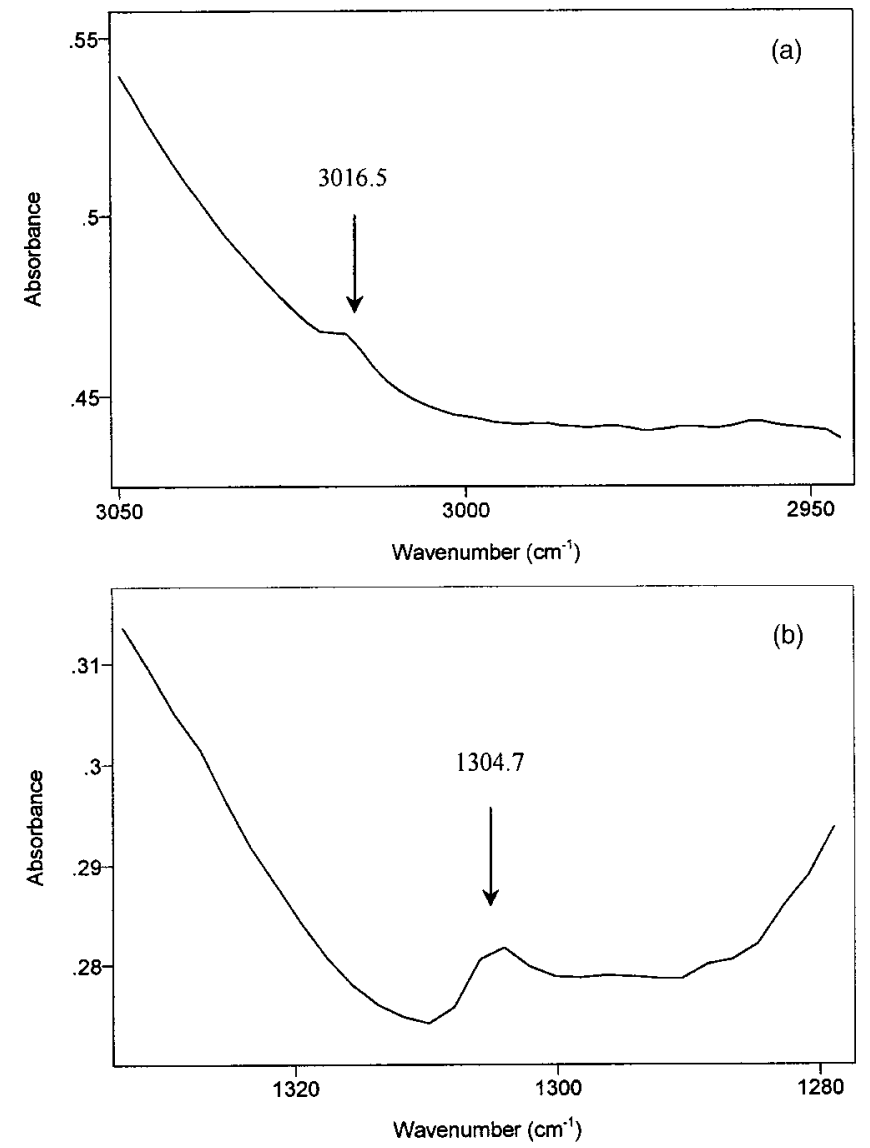

FIG. 2. Double beam spectra of a solution of $\mathrm{CH}_{4}$ in $\mathrm{D}_{2} \mathrm{O}$, using a pure $\mathrm{D}_{2} \mathrm{O}$ single beam spectra as a reference. The spectra were made using a lowtemperature cell $\left(\right.$ at $6{ }^{\circ} \mathrm{C}$ ) fitted with $\mathrm{AgCl}$ windows and a path length of $0.25 \mathrm{~mm}$ : (a) antisymmetric stretching mode $\left(\nu_{3}\right)$ and (b) antisymmetric bending mode $\left(\nu_{4}\right)$.

band intensity is just three times larger than the noise. It is clear that with a $0.123 \mathrm{~mm}$ cell at room temperature the $\nu_{4}$ band intensity would become too close to the noise level, preventing its correct detection. It is also interesting to note that the ratio of the integrated band intensities for $\nu_{3} / \nu_{4}$ is 3.35 in the $\mathrm{D}_{2} \mathrm{O}$ solution and 2.26 for gaseous methane at room temperature. The $\nu_{3} / \nu_{4}$ intensity ratio in gas phase refers to the difference in the absorption coefficients $(A)$ of $\nu_{3}$ and $\nu_{4}$ bands. $A\left(\nu_{3}\right)$ is about two times higher than $A\left(\nu_{4}\right)$, which is in accord with the integrated intensity ratio of the gas phase bands (Table I).

It seems to be more difficult to explain the differences between relative integrated intensities, but it is a common occurrence that the relative intensity of fundamentals is not the same in the gas and condensed states. The present state of the theory for intensities in condensed phases presents greater complications than in the gas phase, which is attributable in part to intermolecular interactions. In addition, the vibrational amplitudes and consequently the induced dipole moments can be different in gas phase and in solutions for stretching and bending modes.

The strong band broadening in the aqueous solution is clearly seen in Table I. Since there is no rotational fine structure in the aqueous solution the $P, Q$, and $R$ lines collapse to form a broad absorption band. It can be concluded that the $\nu_{3}$ 

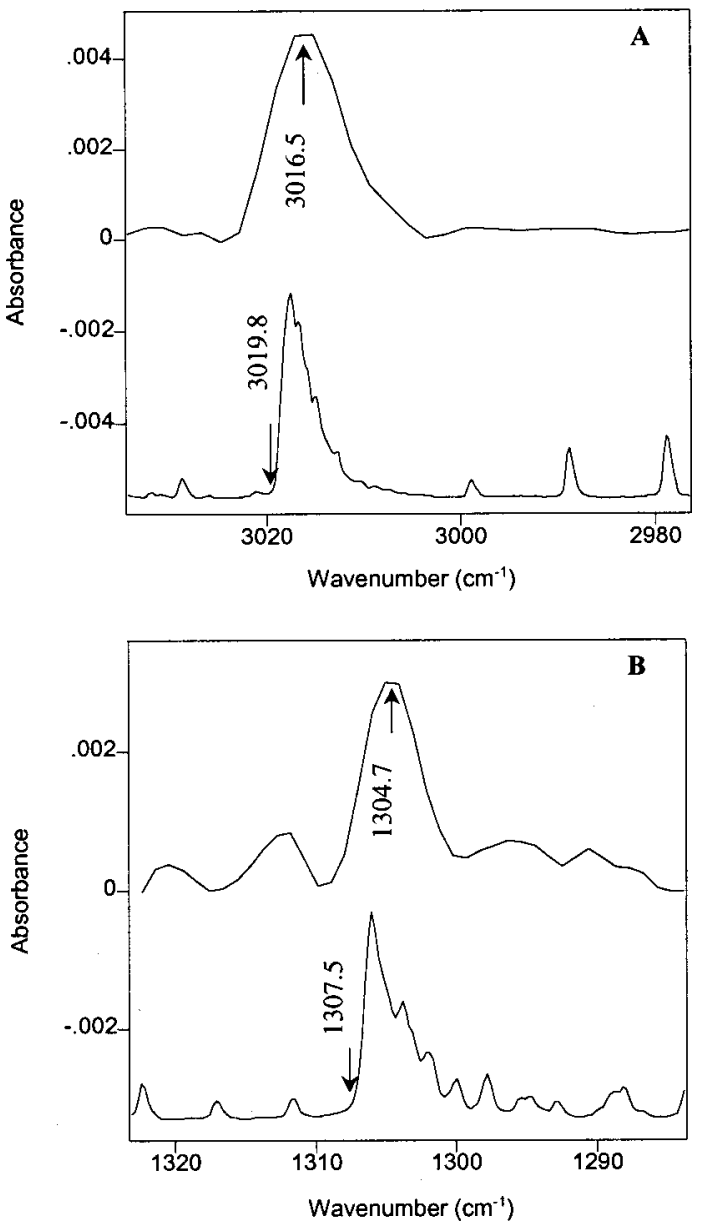

FIG. 3. Baseline corrected bands of a solution of $\mathrm{CH}_{4}$ [top spectra (a) and (b)] in $\mathrm{D}_{2} \mathrm{O}$, produced from the spectra presented in Fig. 2. and part of gas phase $\mathrm{CH}_{4}$ rotation-vibration spectra (bottom features). The positions of the fundamental modes of $\mathrm{CH}_{4}$ in the gas phase and dissolved state are also shown: (a) antisymmetric stretching mode $\left(\nu_{3}\right)$ and (b) antisymmetric bending mode $\left(\nu_{4}\right)$. The absorbance scale refers to the intensity of the bands in aqueous solution.

and $\nu_{4}$ modes undergo a redshift of 3.3 and $2.8 \mathrm{~cm}^{-1}$, respectively, when the methane molecules pass from the gas phase to the solution phase.

\section{B. Molecular dynamics simulations}

The hindered translational and rotational frequency values were obtained from molecular dynamics simulation data using the DL_POLY package ${ }^{19}$ and following various procedures well documented in the literature. ${ }^{6,20}$
We chose to simulate one methane molecule, modeled by the corresponding OPLS-AA (all-atom) set of parameters (five Lennard-Jones centers plus point charges), ${ }^{21}$ surrounded by 299 water molecules, described as TIP-5P particles (one Lennard-Jones center plus point charges). ${ }^{22}$ The watermethane interaction was defined using the Lorentz-Berthelot combining rule to parametrize the cross interactions between Lennard-Jones centers. In order to test the physical consistency of the results, runs with pure $\mathrm{CH}_{4}$ and aqueous solutions of $\mathrm{CD}_{4}$ (based on the same OPLS-AA set and a different total mass) were also performed.

The system was initially equilibrated as an isothermalisobaric $(N p T)$ ensemble until a temperature of $298 \mathrm{~K}(90 \mathrm{~K}$ in the case of pure $\mathrm{CH}_{4}$ ) and a pressure of 1 bar was obtained. Equilibration times ranged from 100 to 500 ps. Each equilibration run was then followed by a series of short runs in the microcanonical ( $N V E$ ) ensemble, with a time step of 2 fs for approximately $500 \mathrm{fs}$. The positional and velocity data from these successive runs were used to calculate the velocity and angular velocity autocorrelation functions (ACFs) of methane in water. Fourier transforms of the ACF yield translational and rotational power spectra from which the external frequencies can be inferred. The translational and rotational frequencies of methane in aqueous solution obtained using this method are included in Table II and are discussed in Sec. IV.

The velocity ACFs for $\mathrm{CH}_{4}$ and for $\mathrm{CD}_{4}$ in aqueous solution at $298 \mathrm{~K}$ and pure $\mathrm{CH}_{4}$ at $90 \mathrm{~K}$ exhibit similar decays, corresponding to translational frequency values of 65,56 , and $50 \mathrm{~cm}^{-1}$, respectively (Fig. 4, top). The ratio of the $\mathrm{CH}_{4}$ and $\mathrm{CD}_{4}$ frequency values is similar to the inverse of the square root ratio of the corresponding masses, $\nu\left(\mathrm{CH}_{4}\right) / \nu\left(\mathrm{CD}_{4}\right)=\left(m\left(\mathrm{CD}_{4}\right) / m\left(\mathrm{CH}_{4}\right)\right)^{1 / 2}$, confirming the physical soundness of the molecular dynamics technique employed. Furthermore, the two velocity ACF curves in aqueous solutions show larger oscillations than the corresponding velocity ACF for pure methane. Apparently the more structured water molecules around methane turn the single cell model approximation into a more realistic model in aqueous solution than in pure liquid methane, as can be confirmed by the better defined peaks in the corresponding power spectra (Fig. 4, top left). It is interesting to note that the analysis of microwave spectra recorded in a molecular beam of $\mathrm{CH}_{4}$ with water yielded a value of $55 \mathrm{~cm}^{-1}$ for the hindered translational frequency of $\mathrm{CH}_{4}$ in the $\mathrm{CH}_{4}-\mathrm{H}_{2} \mathrm{O}$ complex, ${ }^{23}$ which is in good agreement with the frequency values ob-

TABLE I. Infrared absorption bands characteristic of $\mathrm{CH}_{4}$ in $\mathrm{D}_{2} \mathrm{O}$ and in the gaseous state.

\begin{tabular}{|c|c|c|c|c|c|}
\hline System & Band & $\begin{array}{l}\text { Frequency } \\
\left(\mathrm{cm}^{-1}\right)\end{array}$ & $\begin{array}{l}\text { Intensity } \\
(\text { A.U. })^{\mathrm{a}}\end{array}$ & $\begin{array}{l}\text { Band } \\
\text { halfwidth } \\
\left(\mathrm{cm}^{-1}\right)\end{array}$ & $\begin{array}{c}\text { Integrated } \\
\text { intensity } \\
\text { (A.U./cm) }\end{array}$ \\
\hline \multirow{2}{*}{$\begin{array}{c}\mathrm{CH}_{4} \text { in } \mathrm{D}_{2} \mathrm{O} \\
\left(6^{\circ} \mathrm{C}\right)\end{array}$} & $\nu_{3}$ & $3016.5 \pm 0.2$ & 0.0043 & 8.3 & 0.0422 \\
\hline & $\nu_{4}$ & $1304.7 \pm 0.2$ & 0.0027 & 4.7 & 0.0126 \\
\hline Gaseous $\mathrm{CH}_{4}$ & $\nu_{3}$ & 3019.8 & $0.0935^{\mathrm{b}}$ & $3.9^{\mathrm{b}}$ & $1.408^{\mathrm{b}}$ \\
\hline (100 ppm m concentration) & $\nu_{4}$ & 1307.5 & $0.0570^{\mathrm{b}}$ & $3.3^{\mathrm{b}}$ & $0.624^{\mathrm{b}}$ \\
\hline
\end{tabular}

a Absorbance units.

${ }^{\mathrm{b}}$ Band parameters refer to $Q$ branches of $\nu_{3}$ and $\nu_{4}$ bands. 
TABLE II. Vibrational, hindered translational, and rotational frequencies $\nu$ and frequency shifts $\Delta \nu=\nu_{\text {gas }}-\nu_{\text {liq. }}$ of pure methane and methane in aqueous solution (all values in $\mathrm{cm}^{-1}$ ).

\begin{tabular}{|c|c|c|c|c|c|c|c|c|c|c|c|c|}
\hline \multirow[b]{3}{*}{ Mode } & \multicolumn{6}{|c|}{ Pure methane $^{\mathrm{a}}$} & \multicolumn{6}{|c|}{ Methane in aqueous solution } \\
\hline & \multicolumn{3}{|c|}{ Gas, $\nu$} & \multicolumn{3}{|c|}{ Liquid, $\Delta \nu, \nu^{\mathrm{b}}$} & \multicolumn{3}{|c|}{ Gas, $\nu$} & \multicolumn{3}{|c|}{ Liquid, $\Delta \nu, \nu^{\mathrm{b}}$} \\
\hline & $\mathrm{CH}_{4}$ & $\mathrm{CD}_{4}$ & ${ }^{13} \mathrm{CH}_{4}$ & $\mathrm{CH}_{4}$ & $\mathrm{CD}_{4}$ & ${ }^{13} \mathrm{CH}_{4}$ & $\mathrm{CH}_{4}{ }^{\mathrm{a}}$ & $\mathrm{CD}_{4}{ }^{\mathrm{d}}$ & ${ }^{13} \mathrm{CH}_{4}{ }^{\mathrm{d}}$ & $\mathrm{CH}_{4}{ }^{\mathrm{e}}$ & $\mathrm{CD}_{4}{ }^{\mathrm{d}}$ & ${ }^{13} \mathrm{CH}_{4}{ }^{\mathrm{d}}$ \\
\hline Sym.str.(A) & 3143.709 & $2223.791^{\mathrm{c}}$ & 3143.709 & 11.541 & $8.163^{\mathrm{b}}$ & 11.541 & 3143.709 & 2223.791 & 3143.709 & 2.6 & 1.867 & 2.600 \\
\hline Sym.bend. $(E)$ & 1574.199 & 1113.558 & 1574.199 & 3.894 & 2.755 & 3.894 & 1574.199 & 1113.554 & 1574.199 & $\overline{2.3}$ & 1.592 & 2.300 \\
\hline Antisym.str. $(T)$ & 3154.069 & 2333.196 & 3143.048 & 13.840 & 11.480 & 13.645 & 3154.069 & 2338.387 & 3142.137 & $\overline{3.3}$ & 2.544 & 3.277 \\
\hline Antisym.bend. $(T)$ & 1357.423 & 1027.030 & 1348.903 & 4.863 & 2.987 & 4.894 & 1357.423 & 1024.745 & 1348.924 & $\overline{2.8}$ & 1.996 & 2.688 \\
\hline Transl. $(T)$ & - & - & - & 77.693 & 69.451 & 75.37 & - & - & - & 65 & 58.113 & 63.001 \\
\hline Rot. (T) & - & - & - & 72.285 & 51.133 & 72.285 & - & - & - & $\overline{0}$ & 0 & 0 \\
\hline
\end{tabular}

${ }^{\mathrm{a}}$ All values taken from Ref. 27.

${ }^{\mathrm{b}}$ Frequency shifts for the internal vibrations, frequency values for the external modes.

${ }^{c}$ Missing from Ref. 27. Estimated using $\nu\left(\mathrm{CH}_{4}\right) / \nu\left(\mathrm{CD}_{4}\right)=\left(m\left(\mathrm{CD}_{4}\right) / m\left(\mathrm{CH}_{4}\right)\right)^{1 / 2}$.

${ }^{\mathrm{d}}$ Calculated using Wilson's $G F$ matrix method with a Urey-Bradley force field (Table IV).

${ }^{\mathrm{e}}$ Experimental and estimated frequency shifts for the internal modes and translational frequency.

tained from molecular dynamics (MD) simulations.

The angular velocity ACF for $\mathrm{CH}_{4}$ in aqueous solution at $298 \mathrm{~K}$ and for pure $\mathrm{CH}_{4}$ at $90 \mathrm{~K}$ are also presented in Fig. 4. The corresponding power spectra show amplitude maxima at near zero frequency values indicating that, at least according to the molecular potential used for methane, most of the molecules are rotating freely in both condensed phases. Again the peak in aqueous solution is better defined. The ACF curve for $\mathrm{CH}_{4}$ in aqueous solution now shows a monotonous decay, while its pure methane counterpart is very similar to the corresponding velocity ACF (compare Fig. 4 top and bottom). The free rotation of the methane molecules in the pure liquid and aqueous phases is corroborated by recent inelastic neutron scattering studies which showed that $\mathrm{CH}_{4}$ molecules rotate almost freely in the cages of methane hydrates. ${ }^{24}$

Our ACF results can also be compared with the values reported by Laaksonen and Stilbs. ${ }^{20}$ It must be noted that in their study the conditions are slightly different: water and methane are modeled by the earlier TIP4P and united-atom OPLS potentials, respectively, and the aqueous solutions of methane are more concentrated (they contain four methane particles per simulation box). Two major differences are apparent between the two sets of results: (i) Our velocity ACF values show a faster decay in pure methane at $90 \mathrm{~K}$. This can be attributed to a slightly more hindered translational movement of the methane molecules due to the atom-explicit OPLS-AA potential used. The corresponding translational frequency values cannot be compared directly since no power spectra are presented in Ref. 20; and (ii) Our velocity ACF values of both $\mathrm{CH}_{4}$ and $\mathrm{CD}_{4}$ in aqueous solution show larger oscillations. This can be explained again by the differences in the intermolecular potential models employed but also by considering only a single methane molecule in the simulation box. Preliminary tests have shown that in simulation runs with four methane molecules per box, the probabil-
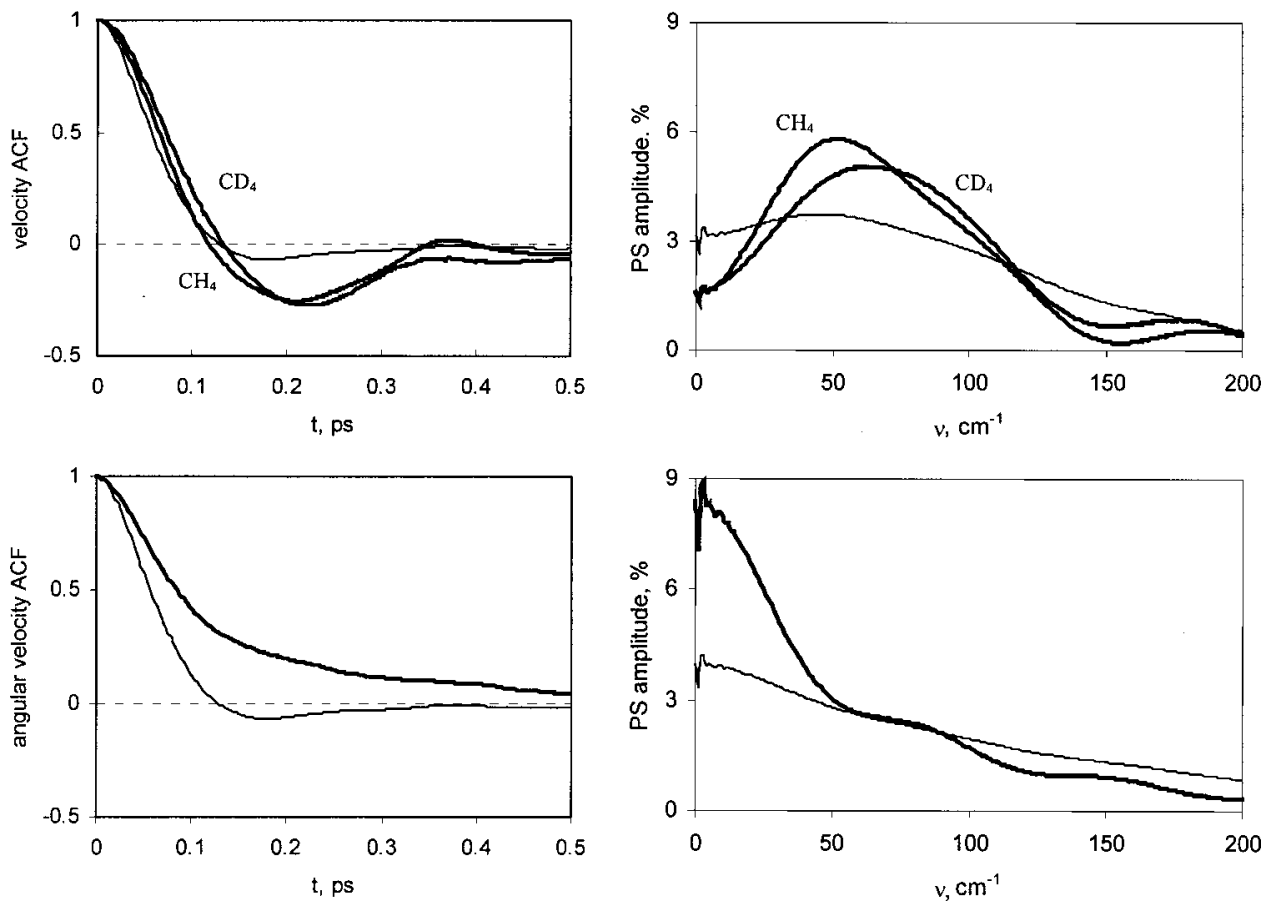

FIG. 4. Translational (top) and angular velocity (bottom) ACFs for methane and the corresponding power spectra (PS). The thin lines correspond to simulations performed with pure $\mathrm{CH}_{4}$ at $90 \mathrm{~K}$; the thick lines to methane $\left(\mathrm{CH}_{4}\right.$ or $\left.\mathrm{CD}_{4}\right)$ in aqueous solution at $298 \mathrm{~K}$. No $\mathrm{CD}_{4}$ data are presented for the angular velocity ACF and corresponding PS. 
TABLE III. Comparisons between theoretical (Calc.) and experimental (Expt.) partition function ratios $\left(f_{\text {liq }} / f_{\text {gas }}\right)$ of pure methane and methane in aqueous solution undergoing either $\mathrm{H} / \mathrm{D}$ or ${ }^{12} \mathrm{C} /{ }^{13} \mathrm{C}$ isotopic substitution. The contributions from the internal (Int.) and external (Ext.) modes and nonclassical rotation (Nclr.) to the overall calculated isotope effect are also shown.

\begin{tabular}{|c|c|c|c|c|c|c|c|c|c|c|c|}
\hline \multirow[b]{2}{*}{$T / \mathrm{K}$} & \multicolumn{5}{|c|}{$10^{3} \cdot \ln \left(f_{\text {liq }} / f_{\text {gas }}\right)$ pure methane } & \multirow[b]{2}{*}{$T / \mathrm{K}$} & \multicolumn{5}{|c|}{$10^{3} \cdot \ln \left(f_{\text {liq }} / f_{\text {gas }}\right.$ methane in aqueous solution } \\
\hline & Int. & Ext. & Nclr. & Calc. & Expt. & & Int. & Ext. & Nclr. & Calc. & Expt. \\
\hline \multicolumn{12}{|c|}{$\mathrm{H} / \mathrm{D}$} \\
\hline 100 & -132.10 & 98.93 & 9.37 & -23.80 & -21.49 & 285 & -16.64 & 2.70 & 3.29 & -10.65 & $-21 \pm 5$ \\
\hline 110 & -120.09 & 81.76 & 8.52 & -29.81 & -26.93 & 298 & -15.90 & 2.47 & 3.14 & -10.29 & $-14 \pm 4$ \\
\hline 120 & -110.09 & 68.70 & 7.81 & -33.57 & -30.34 & 308 & -15.38 & 2.31 & 3.04 & -10.03 & $-13 \pm 4$ \\
\hline & & & & & & 318 & -14.92 & 2.17 & 2.95 & -9.80 & $-17 \pm 5$ \\
\hline 298 & -44.33 & 11.14 & 3.14 & -30.05 & - & 324 & -14.61 & 2.09 & 2.89 & -9.64 & $-18 \pm 5$ \\
\hline \multicolumn{12}{|c|}{${ }^{12} \mathrm{C} /{ }^{13} \mathrm{C}$} \\
\hline 100 & -3.54 & 9.20 & 0.00 & 5.66 & 4.03 & 293 & -0.27 & 0.77 & 0.00 & 0.50 & $0.62 \pm 0.05$ \\
\hline 110 & -3.22 & 7.60 & 0.00 & 4.39 & 2.89 & 323 & -0.24 & 0.63 & 0.00 & 0.39 & $0.44 \pm 0.05$ \\
\hline 120 & -2.95 & 6.39 & 0.00 & 3.44 & 2.06 & 353 & -0.22 & 0.53 & 0.00 & 0.31 & $0.34 \pm 0.05$ \\
\hline 298 & -1.19 & 1.04 & 0.00 & -0.15 & - & & & & & & \\
\hline
\end{tabular}

ity of contact-pair or solvent-separated pair formation for methane is not negligible. This situation leads to stable configurations where the methane molecules experience different types of solvent environment. This is not considered to be a good representation of an infinitely dilute aqueous solution of methane (mole fraction smaller than $10^{-5}$ ). The presence of a single methane molecule per box can affect the (already poor) statistics of the simulation. In order to avoid such problems several independent runs, each with a single methane molecule, were performed and their results were jointly used to provide better statistics.

Our angular velocity ACF curves were not directly compared with the results reported in Ref. 20 as it is not clear how the authors calculated an angular velocity ACF of a methane molecule modeled by an uncharged one-center potential (the united-atom OPLS potential used in runs I, III, IV, and VI of Ref. 20).

\section{RESULTS AND DISCUSSION}

\section{A. Inverse and normal isotope effects in pure methane}

A crude calculation of the solubility isotope effect in aqueous solutions of methane can be performed without the knowledge of the frequency data estimated in Sec. III. As stressed above, both vapor pressure isotope effects of pure substances and isotope effects on the Henry's law coefficients of solutions can be analyzed using the same theoretical framework. In both cases the single-cell model adopted by the theory to characterize the condensed phase can be visualized either as one methane molecule surrounded by other methane molecules or, in the aqueous solution case, as a molecule of methane encircled by water molecules.

The fact that the partial molar volume at infinite dilution of methane in water at $298 \mathrm{~K}$ and the molar volume of pure methane around $90 \mathrm{~K}$ are comparable (around 37 and 36 $\mathrm{cm}^{3} \mathrm{~mol}^{-1}$, respectively $)^{25,26}$ indicates that the size of the "solvent cell" experienced by a methane molecule is similar in both situations. If it is assumed that the interactions between the methane molecule and its surroundings are also alike (mainly London dispersion forces) then the frequency values observed in liquid methane around $90 \mathrm{~K}$ must be comparable with the ones characteristic of methane in the aqueous solutions at $298 \mathrm{~K}$. This "similar solvent cell" assumption finds a semiquantitative justification not only in the partial molar volume data but also in the simulation results discussed in the previous section.

If this hypothesis is considered, then all spectroscopic data needed to calculate the solute isotope effect can be taken from previous studies of vapor pressure isotope effects in pure methane. ${ }^{27}$

The relevant data are compiled in Table II and Eqs. (2) and (3) were used to extrapolate the isotope effect to temperatures around $298 \mathrm{~K}$. The results are presented in Table III and in Fig. 5.

Although the deviations form the experimental solubility isotope effect values are substantial in both cases, the order of magnitude and sign of the H/D solute isotope is predicted correctly. This is an encouraging result taking into account the simplicity of the assumptions made and the extent of the extrapolation involved. In other words, the inverse solubility isotope effect of methane in water can be understood, at least semiquantitatively, using the same arguments that explain the inverse vapor pressure isotope effect in the pure methane at lower temperatures: the predominance of the frequency redshifts of the internal modes when methane molecules are moved from the gas to the condensed phase. ${ }^{27}$ Redshifts on condensation are the general rule as internal vibrations are concerned-since the number of intermolecular interactions is larger in condensed phases, the strength of the intramolecular modes is smaller. In the case of methane undergoing H/D substitution, the redshifts from the internal modes are large enough to overcome the contribution from the external (translational) modes.

\section{B. Solubility isotope effects evaluated from spectroscopic data in aqueous solution}

The precision of the estimation of the solubility isotope effect can be improved if the redshifts of the vibrational frequencies of $\mathrm{CH}_{4}$ in aqueous solution obtained experimentally and the molecular dynamics data concerning translational 

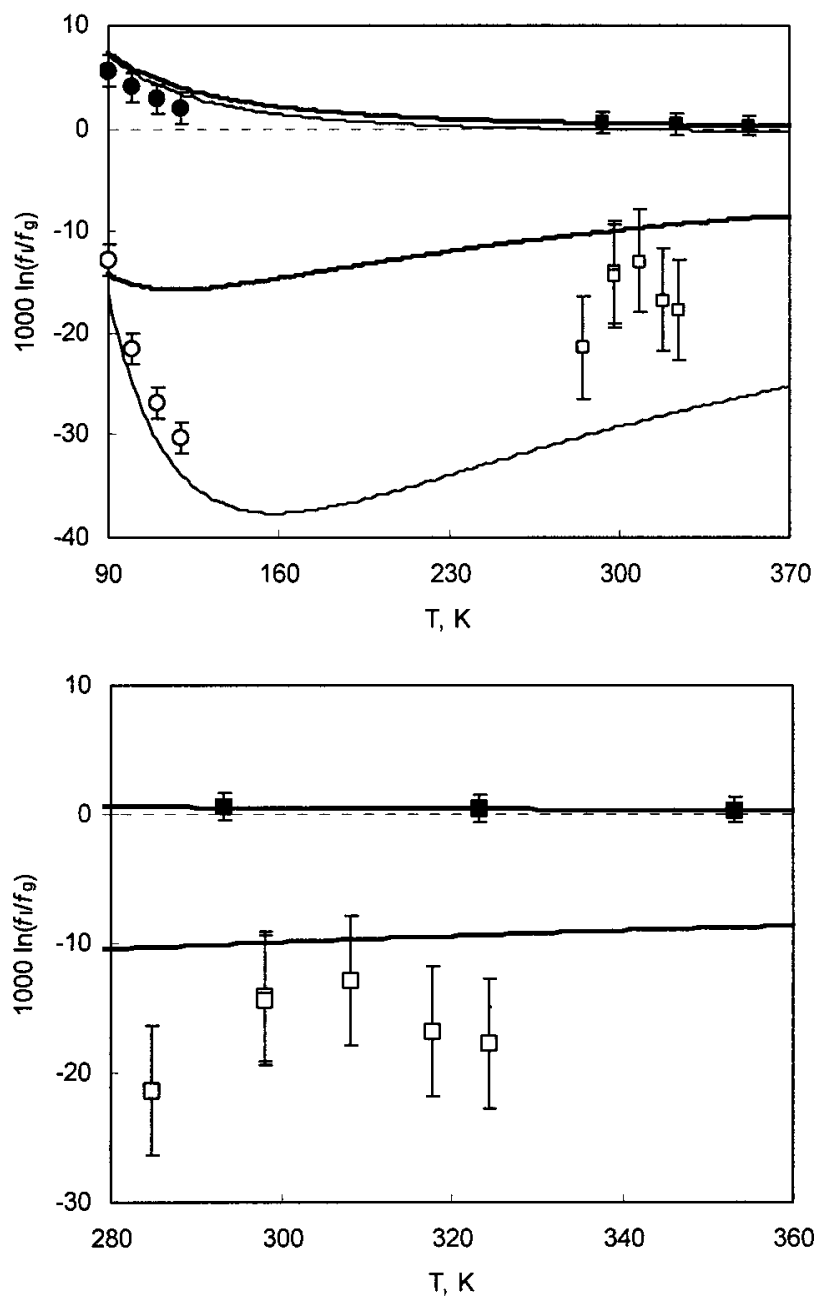

FIG. 5. Isotope effects of methane undergoing $\mathrm{H} / \mathrm{D}$ or ${ }^{12} \mathrm{C} /{ }^{13} \mathrm{C}$ substitution as a function of temperature. The circles correspond to experimental vapor pressure isotope effect data in pure methane at cryogenic temperatures: $(\bigcirc)$ $\mathrm{H} / \mathrm{D}$ substitution effect; $(\bullet){ }^{12} \mathrm{C} /{ }^{13} \mathrm{C}$ substitution. The squares correspond to the experimental isotope effect on the Henry's law coefficients data in aqueous solutions of methane around room temperature: ( $\square$ ) H/D substitution; (ם) ${ }^{12} \mathrm{C} /{ }^{13} \mathrm{C}$ substitution. The thin lines represent the theoretical estimation of the vapor pressure isotope effect (Sec. IV A) and are extrapolated from cryogenic temperatures to room temperature. The thick lines represent the theoretical estimation of the isotope effect on the Henry's law coefficients around room temperature (Sec. IV B) and are extrapolated to cryogenic temperatures. Lines representing positive ("normal") isotope effects correspond to ${ }^{12} \mathrm{C} /{ }^{13} \mathrm{C}$ substitution, while their negative ("inverse") counterparts represent $\mathrm{H} / \mathrm{D}$ substitution.

and rotational motions of methane in water are considered. The IR spectroscopic measurements yielded frequency redshifts of 3.3 and $2.8 \mathrm{~cm}^{-1}$, for the antisymmetric stretching and bending modes, respectively. On the other hand the MD simulations predicted a frequency value of $65 \mathrm{~cm}^{-1}$ for the hindered translation of $\mathrm{CH}_{4}$ in aqueous solution and free rotation of the molecule (Table II).

The experimental frequency shifts of the IR inactive symmetric modes for $\mathrm{CH}_{4}$ in aqueous solution are still missing. They were estimated considering for each mode the ratio between symmetric and antisymmetric experimental vaporliquid frequency shifts for pure liquid methane, ${ }^{27}$ $\left(\Delta \nu_{\text {sym. }} / \Delta \nu_{\text {antisym. }}\right)_{\left.\mathrm{CH}_{4} \text { (aq.) }\right)} \cong\left(\Delta \nu_{\text {sym. }} / \Delta \nu_{\text {antisym. }}\right)_{\mathrm{CH}_{4}(l)}$, yielding values of 2.6 and $2.3 \mathrm{~cm}^{-1}$ for the frequency redshifts of the symmetric stretching and bending modes, respectively (Table II).

The frequency values of the internal modes for the other isotopic methane species $\left(\mathrm{CD}_{4}\right.$ and $\left.{ }^{13} \mathrm{CH}_{4}\right)$ were calculated using Wilson's $G F$ matrix method. ${ }^{17}$ Since all methane molecules involved in this work are spherical tops, the most convenient way to set up the force constant matrices was to employ the Urey-Bradley force field and the corresponding $G$ matrices $^{28}$ (Table IV). The four distinct force constants needed to set up the $F$ matrices for the gas and aqueous phases were calculated from vibrational frequency values of $\mathrm{CH}_{4}$ presented in Table II. The $F$ matrices are isotope independent and along with the three $G$ matrices defining each isotopomer permit, through the solution of the corresponding secular equation, the calculation of the six frequency (eigenvalue) sets characterize the internal vibrational motions of each isotopomer in each phase. The calculated frequencies are listed in Table II, whereas the $G F$ matrices are compiled in Table IV. No coupling between external and internal modes was considered in the calculations.

The hindered translational frequencies of $\mathrm{CD}_{4}$ and ${ }^{13} \mathrm{CH}_{4}$ in water were estimated using the value for $\mathrm{CH}_{4}$ and the square root of the appropriate ratio between isotopomer masses, $\quad \nu_{\mathrm{CD}_{4}}=\nu_{\mathrm{CH}_{4}}\left(m_{\mathrm{CH}_{4}} / m_{\mathrm{CD}_{4}}\right)^{1 / 2}$ and $\nu_{13 \mathrm{CH}_{4}}$ $=\nu_{\mathrm{CH}_{4}}\left(m_{\mathrm{CH}_{4}} / m_{13 \mathrm{CH}_{4}}\right)^{1 / 2}$. The rotation of methane in aqueous solution was taken as unhindered. The frequency values of the external modes for all isotopic species are also listed in Table II.

The contribution of the nonclassical rotation to the reduced partition function in the gas phase was estimated using the expression valid for spherical tops ${ }^{16}$

$$
\ln f_{\mathrm{nclr}}=\left(\frac{1}{I_{\mathrm{CD}_{4}}}-\frac{1}{I_{\mathrm{CD}_{4}}}\right)\left(\frac{\hbar^{2}}{4 k_{b} T}\right)
$$

where $I_{i}$ represents the moment of inertia of species $i$ and $\hbar=h / 2 \pi$.

The reduced partition function ratios for the gas and aqueous phases were calculated as a function of temperature and for each type of isotopic substitution $\left(\mathrm{H} / \mathrm{D}\right.$ or $\left.{ }^{12} \mathrm{C} /{ }^{13} \mathrm{C}\right)$ and are presented in Table III and Fig. 5 for temperatures matching the experimental results.

The ${ }^{12} \mathrm{C} /{ }^{13} \mathrm{C}$ solubility isotope effect of methane is predicted in good agreement with the experimental results. In this case only the antisymmetric internal modes and the hindered translation in the aqueous phase contribute to the overall isotope effect and all quantities were determined either experimentally (IR results) or by MD simulation. Even the large uncertainty associated with assigning a frequency value to the hindered translational motion (the peaks in the translational power spectra are broadbands) does not affect the order of magnitude of the predicted results.

The sign and order of magnitude of the H/D solubility isotope effect is predicted correctly but the calculated values (an inverse isotope effect around 1.0\%) underestimate the experimental data. The change in the calculated inverse isotope effect with increasing temperature is very small, which is in agreement with the experiment if one considers the imprecision of the experimental data. 
TABLE IV. $F$ and $G$ matrix elements for the calculation of the internal frequency values (eigenvalues) of methane in the gas and aqueous solution using the Urey-Bradley force field. ${ }^{a}$

\begin{tabular}{|c|c|c|c|c|c|c|c|}
\hline \multicolumn{5}{|c|}{$G$ matrix elements of a molecule $\mathrm{AX}_{4}$} & \multicolumn{3}{|c|}{ Atomic mass (a.u.) } \\
\hline A & $\mu_{X}$ & 0 & 0 & 0 & & $1 / \mu(\mathrm{H})$ & 1.0078246 \\
\hline $\mathrm{E}$ & 0 & $3 \mu_{X}$ & 0 & 0 & & $1 / \mu(\mathrm{D})$ & 2.0141022 \\
\hline $\mathrm{T}$ & 0 & 0 & $\mu_{X}+4 \mu_{A} / 3$ & $-8 \mu_{A} / 3$ & & $1 / \mu\left({ }^{12} \mathrm{C}\right)$ & 12 \\
\hline $\mathrm{T}$ & 0 & 0 & $-8 \mu_{A} / 3$ & $2 \mu_{X}+16 \mu_{A} / 3$ & & $1 / \mu\left({ }^{13} \mathrm{C}\right)$ & 13.033554 \\
\hline & \multicolumn{4}{|c|}{$F$ matrix elements of a molecule $\mathrm{AX}_{4}$} & \multicolumn{3}{|c|}{ Force constants $(\mathrm{N} / \mathrm{m})$} \\
\hline & & & & & & Gas & Solution \\
\hline A & $K+4 F$ & 0 & 0 & 0 & K & 5.04503 & 5.033335 \\
\hline $\mathrm{E}$ & 0 & $H-F^{\prime} / 3+F / 3$ & 0 & 0 & $H$ & 0.430439 & 0.42875 \\
\hline $\mathrm{T}$ & 0 & 0 & $K+4\left(F^{\prime}+F\right) / 3$ & $2\left(F^{\prime}+F\right) / 3$ & $F^{\prime}$ & 0.025681 & 0.025782 \\
\hline $\mathrm{T}$ & 0 & 0 & $2\left(F^{\prime}+F\right) / 3$ & $\mathrm{H}-\left(F-5 F^{\prime}\right) / 3$ & $F$ & 0.205738 & 0.205304 \\
\hline
\end{tabular}

${ }^{\mathrm{a}}$ See Ref. 28.

One source of uncertainty in the model calculation is the estimation of the frequency shifts for the symmetric stretching and bending modes of methane in the aqueous solution. For example, if these are taken as equal to the shifts of the antisymmetric modes, the calculated isotope effects are more consistent with the experiment.

If the uncertainties associated with both the experimental determination of the Henry's law coefficients and the measurement of the frequency redshifts are considered, we conclude that both sets indicate an inverse solubility isotope effect between $1 \%$ and $2 \%$ for the H/D substitution.

\section{CONCLUSION}

The first conclusion that can be drawn from the present work is the possibility of interpreting the isotope effect on the Henry's law coefficients using the same theoretical framework as developed for the well-studied vapor pressure isotope effects. In other words the statistical mechanical theory of condensed phase isotope effects can treat solubility isotope effects in solution as a particular case of isotope effects in vapor-liquid equilibrium.

In the first part of the discussion the similarity between vapor pressure isotope effects and solubility isotope effects was explored and led to a crude estimation of the solubility isotope effect of methane in water using spectroscopic data taken from a study of pure methane at a completely different temperature range. The inverse isotope effect found in both cases can be interpreted in an analogous way and the calculations even with the crude assumptions involved yielded semiquantitatively correct results.

The statistical mechanical theory of condensed phase isotope effects is based on the evaluation of the reduced partition function ratios for each phase at equilibrium. This requires the knowledge of spectroscopic data characterizing each molecular motion in both phases. In the case of gases dissolved in water close to infinite dilution data of this type are generally not available. In the present paper a concerted effort was undertaken to obtain either experimentally or by molecular simulation some of the missing values. It seems as though some of the spectroscopic data (for instance the frequency of the hindered translational motion of methane in water) can only be estimated using molecular simulation methods which will probably be a route of choice in the interpretation of isotope effects in solution. ${ }^{6}$

The solubility isotope effects in the Henry's law coefficients represent small differences between large numerical values. Furthermore, in the case of $\mathrm{H} / \mathrm{D}$ and ${ }^{12} \mathrm{C} /{ }^{13} \mathrm{C}$ substitution of methane in aqueous solution, the solubility isotope effects have different orders of magnitude $(-1.6 \%$ and $+0.05 \%$, respectively). Still the data were accessed with the required precision both experimentally (using different techniques) and theoretically. This effort seems to be justified by the growing importance of this type of effect in the treatment of biological and geological problems reinforcing the significance of a correct theoretical interpretation of existing experimental data.

\section{ACKNOWLEDGMENTS}

One of the authors (G.J.) wishes to thank the Hungarian Research Fund, OTKA, Grant No. T031829 for financial support. The collaboration between the French and Portuguese investigation teams was supported by the CNRS/ ICCTI cooperation program (Project Nos. 8839 and 423, respectively). The authors (Z.B. and J.M.) express their thanks to the Hungarian Ministry of Education (Grant No. FKFP0170/2000) for supporting their gas spectroscopy research.

${ }^{1}$ A. M. Rouhi, Chem. Eng. News 38, 42 (1997).

${ }^{2}$ J. C. Nesheim and J. D. Lipscomb, Biochemistry 35, 10240 (1996).

${ }^{3}$ K. E. Liu and S. J. Lippard, Adv. Inorg. Chem. 42, 263 (1995).

${ }^{4}$ G. Jancsó, Nukleonika 47, S53 (2002).

${ }^{5}$ G. Jancsó and W. A. Van Hook, Chem. Rev. 74, 689 (1974).

${ }^{6}$ L. X. Dang, P. Bopp, and M. Wolfsberg, Z. Naturforsch., A: Phys. Sci. 44, 485 (1989).

${ }^{7}$ P. Bopp and M. Wolfsberg, J. Solution Chem. 10, 357 (1981).

${ }^{8}$ M. F. Costa Gomes and J.-P. Grolier, Phys. Chem. Chem. Phys. 3, 1047 (2000).

${ }^{9}$ B. B. Benson and D. Krause, Jr., J. Solution Chem. 9, 859 (1980).

${ }^{10}$ J. Muccitelli and W.-Y. Wen, J. Solution Chem. 7, 257 (1978).

${ }^{11}$ B. B. Benson, D. Krause, Jr., and M. A. Peterson, J. Solution Chem. 8, 655 (1979).

${ }^{12}$ P. Harting, H. Shütze, and G. Christoph, Isotopenpraxis 12, 232 (1976).

${ }^{13}$ J. Bigeleisen, J. Chem. Phys. 34, 1485 (1961).

${ }^{14}$ J. Bigeleisen and M. G. Mayer, J. Chem. Phys. 15, 261 (1947).

${ }^{15}$ M. J. Stern, W. A. Van Hook, and M. Wolfsberg, J. Chem. Phys. 39, 3179 (1963). 
${ }^{16} \mathrm{G}$. Herzberg, Infrared and Raman Spectra of Polyatomic Molecules (Van Nostrand, New York, 1945).

${ }^{17}$ B. Wilson, Jr., J. C. Decius, and P. C. Cross, Molecular Vibrations (McGraw-Hill, New York, 1955).

${ }^{18}$ L. H. Jones and R. S. McDowell, J. Mol. Spectrosc. 3, 632 (1939).

${ }^{19} \mathrm{~W}$. Smith and T. R. Foster, The DL_POLY Package of Molecular Simulation Routines, Version 2.12 (The Council for the Central Laboratory of Research Councils, Daresbury Laboratory, Daresbury, UK, 1999).

${ }^{20}$ A. Laaksonen and P. Stilbs, Mol. Phys. 74, 747 (1991).

${ }^{21}$ G. Kaminsky, E. M. Duffy, T. Matsui, and W. L. Jorgensen, J. Phys. Chem. 98, 13077 (1994).
${ }^{22}$ M. W. Mahoney and W. L. Jorgensen, J. Chem. Phys. 112, 8910 (2000).

${ }^{23}$ R. D. Suenram, G. T. Fraser, F. J. Lovas, and Y. Kawashima, J. Chem. Phys. 101, 7230 (1994).

${ }^{24}$ C. Gutt, B. Asmussen, and W. Press, J. Chem. Phys. 113, 4713 (2000).

${ }^{25}$ J. H. Hildebrand and R. L. Scott, The Solubility of Nonelectrolytes, 3rd ed. (Reinhold, New York, 1950).

${ }^{26}$ R. Kleinrahm and W. Wagner, J. Chem. Thermodyn. 18, 739 (1986).

${ }^{27}$ J. Bigeleisen, C. B. Cragg, and M. Jeevanandam, J. Chem. Phys. 47, 4335 (1967).

${ }^{28}$ T. Simanouti, J. Chem. Phys. 17, 245 (1949). 